Pacific Journal of Mathematics

ORDERED GLEASON PART

Vol. 62, No. 2

February 1976 


\title{
ORDERED GLEASON PARTS
}

\author{
H. S. BEAR
}

On the points of the domain of a function space we define a partial ordering which extends naturally to the Gleason parts. A general maximum principle and Harnack convergence theorem are proved in terms of the ordering, as well as an integral kernel representation for Dirichlet spaces. The results are applied to a space of solutions of the heat equation, and a space of solutions of the wave equation.

1. Introduction. Gleason parts for function algebras were introduced in [8]. The concept was extended to function spaces by the author in [1], and further studied in [2], [3], [5], [6], [7]. In studying function algebras, or abstract potential theory, one commonly assumes the existence of just one nontrivial part, mirroring the usual situation in algebras of analytic functions, or spaces of harmonic functions. For other function spaces - for example solutions of the heat equation or wave equation - there are infinitely many parts, and the relationship between parts becomes important.

In this paper we introduce a partial ordering on the points of the domain of a function space, and this ordering extends naturally to the parts. We obtain a strong maximum principle and a Harnack convergence theorem in a completely general setting, in terms of the ordering of the domain. The integral kernel representation of [5] also extends to the many-part setting with the appropriate adjustments.

In section four we interpret our results for a space of solutions of the heat equation, where the nontrivial parts form a simply ordered set. The integral kernel representation in this case is a simplified and strengthened version of that given by [4].

Finally, in section five we specialize our results to a space of solutions of the wave equation. We obtain for these functions a maximum principle and Harnack convergence theorem as corollaries of the general results.

The author would like to express his appreciation to Gerald Hile for many helpful comments on this work.

2. The ordering. Let $X$ be a compact Hausdorff space and $B$ a linear space of continuous real functions on $X$. Assume that $B$ contains the constant functions and separates the points of $X$. We give $B$ the sup-norm, and let $B^{*}$ be the dual space, with the $w^{*}$ topology. The space $X$, realized as evaluation functionals, is 
homeomorphic to a subset of the unit ball of $B^{*}$, and $B$ is naturally isomorphic to the space of all $w^{*}$-continuous linear functionals on $B^{*}$. We henceforth regard $X$ as a subset of $B^{*}$. Let $T$ be the closed convex hull of $X$ in $B^{*}$, and let $\Gamma$ be the closure of the extreme points of $T$. Then $T$ is compact, and $\Gamma \subset X \subset T$. For $p \in B^{*}, p \in T$ if and only if $u(p) \leqq \max u[\Gamma]$ for all $u \in B$. Hence $B$ is isometric to the restrictions to $\Gamma$ or $X$ or $T$ of the continuous functionals on $B^{*}$, and we henceforth regard $B$ as a space of linear functions on $T$. The set $\Gamma$ is the Shilov boundary of $B$ in $X$ or $T$.

Definition. For distinct points $p, q$ of $T$ (and hence for $p, q \in X$ ), we write $q \leqslant p$ if and only if the segment from $q$ to $p$ in $B^{*}$ extends beyond $p$ in $T$.

Hence $q \leqslant p$ if and only if $p+\alpha(p-q)=r \in T$ for some $\alpha>0$. In this case we say that $[q, p]$ extends by $\alpha$. (This condition differs from that of [6], in that it is not symmetric in $q$ and $p$.) Clearly $[q, p]$ extends by $\alpha$ if and only if $p=[\alpha /(1+\alpha)] q+[1 /(1+\alpha)] r$ for some $r \in T$. We write $q<p$ if $q \leqslant p$ but not $p \leqslant q$, and $p \sim q$ if $q \leqslant p$ and $p \leqslant q$. We agree that $p \leqslant p$ for all $p$.

THEOREM 1 (Harnack condition). For $q, p \in T ; q \leqslant p$ if and only if there is a number $M \geqq 1$ such that $u(q) \leqq M u(p)$ for all nonnegative $u \in B$. Specifically, $[q, p]$ extends by $\alpha$ if and only if $u(q) \leqq$ $[(\alpha+1) / \alpha] u(p)$ for all $u \geqq 0$.

Proof. Since $T$ is a compact convex set in $B^{*}$, and $B$ is the dual of $B^{*}, r \in T$ if and only if $u(r) \geqq 0$ for all nonnegative $u \in B$. Here we need the fact that constants are in $B$ (cf. [1], [6]). Hence $p+\alpha(p-q)=$ $(1+\alpha) p-\alpha q \in T$ if and only if $(1+\alpha) u(p) \geqq \alpha u(q)$ for all $u \geqq 0$.

If $q \leqslant p$, we let $M(q, p)$ be the minimum $M$ such that $u(q) \leqq M u(p)$ for all $u \geqq 0$. Equivalently, $M(q, p)=(\alpha+1) / \alpha$ for the maximum $\alpha$ such that $[q, p]$ extends by $\alpha$.

COROllaRy 1 . If $r \leqslant q \leqslant p$, then $M(r, p) \leqq M(r, q) M(q, p)$. If $[r, q]$ extends by $\alpha$ and $[q, p]$ by $\beta$, then $[r, p]$ extends by $\gamma$, where $(1+\gamma) / \gamma=(1+\alpha)(1+\beta) / \alpha \beta$.

Proof. The first statement is clear and the second follows from the first. A geometric proof of the corollary appears in [6, Lemma 1].

COROLLARY $2 . \leqslant$ is transitive, and $\sim$ is an equivalence relation. 
Definition (cf. [1], [6]). The equivalence classes of $\sim$ are the Gleason parts of $T$ with respect to $B$. The sets $P \cap X$, for the parts $P$ of $T$, are the Gleason parts of $X$.

The relation $\leqslant$ extends in a natural way to the parts of $T$ or $X: Q \leqslant P$ if and only if $q \leqslant p$ for some (all) $q \in Q$ and $p \in P$. For parts $P$ and $Q, P=Q$ if and only if $P \leqslant Q$ and $Q \leqslant P$.

The following theorem shows that a Harnack convergence theorem holds generally for function spaces. The classical Harnack convergence theorem for harmonic functions is the special case in which $X-\Gamma$ consists of one part.

THEOREM 2 (Harnack convergence theorem). If $u_{n} \in B$ and $u_{n} \leqq$ $u_{n+1}$ for all $n$, and $\left\{u_{n}(p)\right\}$ converges, then $\left\{u_{n}(q)\right\}$ converges for all $q \leqslant p$.

Proof. Let $v_{n}=u_{n}-u_{1}$, so that $v_{n} \geqq 0$ and $\left\{v_{n}(p)\right\}$ is bounded above by some $K$. If $q \leqslant p$, then $v_{n}(q) \leqq M(q, p) v_{n}(p) \leqq K M(q, p)$ for all $n$. Hence $\left\{v_{n}(q)\right\}$, and $\left\{u_{n}(q)\right\}$, converge.

Every function space of course satisfies a weak maximum principle, which is just the statement that the Shilov boundary exists. The next theorem shows that every function space also satisfies a strong maximum principle. The theorem below specializes to the result of [2] for one-part spaces.

TheOREM 3 (Maximum principle). If $u(p)=\max u$, then $u(q)=$ $u(p)$ for all $q \leqslant p$.

Note. The points $p, q$ of interest will be points of $X$ rather than $T-X$, but there is no need to make the distinction in the theorem. It may of course happen that there is no $q$ in $X$ with $q \leqslant p$.

Proof. Observe that $\max u=\max u[T]=\max u[X]=\max u[\Gamma]$. Assume that $u(p)=\max u$, and $u(q)<u(p)$ for some $q \leqslant p$. Then $[q, p]$ extends to some $r \in T$. Since $u$ is linear on $T, u(r)>u(p)$, a contradiction.

Corollary. Any maximum set is a union of parts.

It is easy to see that the following conditions on $p$ and $q$ are equivalent:

(i) for all $u \in B$, if $u(p)=\max u$, then $u(q)=u(p)$;

(ii) for all $u \in B$, if $u(p)=\min u$, then $u(q)=u(p)$;

(iii) for all $u \in B$, if $u \geqq 0$ and $u(p)=0$, then $u(q)=u(p)$. 
We will refer to any of the above as a maximum principle, and say that $p$ maximizes $q$. Theorem 3 says that $p$ maximizes $q$ if $q \leqslant p$.

Definition. A representing measure for a point $p$ of $T$ is a positive Baire probability measure $m_{p}$ on $\Gamma$ such that $u(p)=\int u d m_{p}$ for all $u \in B$.

The following theorem gives a characterization of the ordering $q \leqslant p$ in terms of representing measures.

THEOREM 4. If $q \leqslant p$, then there are representing measures $m_{q}$ and $m_{p}$ such that $m_{q}(E) \leqq M(q, p) m_{p}(E)$ for every measurable set $E$. If $m_{q}=k m_{p}$ for an essentially bounded measurable function $k$, then $q \leqslant p$ and $M(q, p) \leqq$ ess. sup. $f$.

Proof. Assume $q \leqslant p$, and let $[q, p]$ extend by $\alpha$, where $\alpha$ is maximum. Then $M(q, p)=(\alpha+1) / \alpha$, and $p=[1 / M(q, p)] q+$ $[1-1 / M(q, p)] r$ for some $r \in T$. If $m_{q}$ and $m_{r}$ are any representing measures for $q$ and $r$, then $m_{p}=[1 / M(q, p)] m_{q}+[1-1 / M(q, p)] m_{r}$ is a representing measure for $p$, since the functions $u$ of $B$ are linear on $T$. Clearly $m_{q} \leqq M(q, p) m_{p}$. Conversely, if $m_{q}=k m_{p}$, and $u \geqq 0$, then $u(q)=\int u k d m_{p} \leqq($ ess. sup. $k) u(p), \quad$ and $\quad q \leqslant p \quad$ with $\quad M(q, p) \leqq$ ess. sup. $k$.

Notice that we have actually shown that if $q \leqslant p$, then for any representing measure $m_{q}$ there is a representing measure $m_{p}$ such that $m_{q} \leqq M(q, p) m_{p}$.

Definition. $B$ is a Dirichlet space if $B \mid \Gamma$ is uniformly dense in $C(\Gamma)$.

For a Dirichlet space, the representing measures $m_{p}$ are unique, and we have the following characterization of the maximum principle in terms of the measures. We denote the support of $m_{p}$ by $s p m_{p}$.

THEOREM 5. If $B$ is a Dirichlet space, then $p$ maximizes $q$ if and only if $\operatorname{sp} m_{q} \subset \operatorname{sp} m_{p}$.

Proof. For any $u, u(p)=\max u$ if and only if $u$ is constant on sp $m_{p}$. If $p$ maximizes $q$, then $u$ is constant on $s p m_{q}$ whenever $u$ is constant on $s p m_{p}$. Since $B \mid \Gamma$ is uniformly dense in $C(\Gamma)$, this means that $s p m_{q} \subset s p m_{p}$. 
In section four we consider a space of solutions to the heat equation, and in section five a space of solutions of the wave equation. In both these examples the ordering $q \leqslant p$ is essentially equivalent to the fact that $p$ maximizes $q$. This is also true, of course, for any one-part space, such as a space of harmonic functions on a connected set. The following example shows that this is not true in general, even for Dirichlet spaces, without further assumptions.

Example. Let $\Gamma=[0,1]$, and $B=C(\Gamma)$. Then $T$ is all positive probability measures on $\Gamma$, and we let $X=T$. For $u \in B$ and $p \in T$, $u(p)=\int u d p$. The topology in $T$ is pointwise convergence on $B$. Since $B$ is a Dirichlet space, $p$ maximizes $q$ is equivalent to $s p q \subset s p p$. Clearly there are measures $q$ and $p$ such that $s p q \subset s p p$, but $d q / d p$ is not bounded, and hence $q \leqslant p$ fails.

Although the above example shows that one can not prove in general that " $p$ maximizes $q$ " implies $q \leqslant p$, it is not a very satisfying reflection of the situation one is likely to encounter in a "real" example. For a space of solutions of a P.D.E., for example, $X$ would be a compact set in Euclidean space, and a very thin subset of $T$. The $w^{*}$-convergence in $T$ would in fact be metrizable in $X$, and the mapping $p \rightarrow m_{p}$ might well be analytic in one or more of the coordinates of $p$. It would be valuable to know when the maximum principle for $p$ and $q$ does coincide with $q \leqslant p$ in view of the many maximum theorems which hold for solutions of differential equations (see [15]).

3. An integral kernel. For points $p, q$ in the same part $P$ of $T$ on $X$ we let $d(p, q)=\log \min \{M(p, q), M(q, p)\}$. Then $d$ is a complete metric on $P$, and the $d$-topology is stronger than the given topology $[\mathbf{5} ; \mathbf{6}$; $3 \mathrm{Sec}$. 9]. In [5] we developed an integral kernel representation for one-part Dirichlet spaces where the topology on the part is given by $d$. The results of [5] do not apply to many-part spaces, since parts are separated in the $d$-topology. We now define a new metric $D$ on $X-\Gamma$ and obtain the integral representation of [5] in the many-part case when the given topology coincides with the $D$-topology.

Definitions. $X\left(p_{0}\right)=\left\{q \in X: q \leqslant p_{0}\right\} ; B^{+}\left(p_{0}\right)=\{u \in B: u>0$ and $\left.u\left(p_{0}\right)=1\right\}$. For $p, q \in X\left(p_{0}\right)$, let $D(p, q)=\sup \{|u(p)-u(q)|$ : $\left.u \in B^{+}\left(p_{0}\right)\right\}$. Let $\mathscr{T}$ be the given topology of $X$ relativized to $X\left(p_{0}\right)$, and $\mathscr{T}_{D}$ the $D$-topology on $X\left(p_{0}\right)$.

In this section it is more natural to consider subsets of $X$ rather than $T$, and involves no loss of generality. We remark that for spaces of 
parabolic functions $(\S 4)$ there are maximal points $p_{0}$ so that $X\left(p_{0}\right)=$ $X-\Gamma$.

THEOREM 6. $D$ is a complete metric on $X\left(p_{0}\right)$, and $\mathscr{T}_{D}$ is stronger than $\mathscr{T} . \mathscr{T}_{D}=\mathscr{T}$ if and only if $B^{+}\left(p_{0}\right)$ is equicontinuous on $X\left(p_{0}\right)$.

Proof. We think of the points $p \in X\left(p_{0}\right)$ as functions on $B^{+}\left(p_{0}\right)$. Since $u(p) \leqq M\left(p, p_{0}\right) u\left(p_{0}\right)=M\left(p, p_{0}\right)$ for all $u \in B^{+}\left(p_{0}\right)$, the "functions" $p \in X\left(p_{0}\right)$ are bounded on $B^{+}\left(p_{0}\right)$. The metric $D$ is the metric of uniform convergence of the functions $p$ on the set $B^{+}\left(p_{0}\right)$. If $\left\{p_{n}\right\}$ is a $D$-Cauchy sequence in $X\left(p_{0}\right)$, then clearly there is a bounded function $\varphi$ on $B^{+}\left(p_{0}\right)$ such that $u\left(p_{n}\right) \rightarrow \varphi(u)$ uniformly for $u \in B^{+}\left(p_{0}\right)$. To show that $\varphi(u)=u(p)$ for some $p \in X\left(p_{0}\right)$, we pick a subnet $\left\{p_{n_{j}}\right\}$ which $\mathscr{T}$-converges to some $p \in X$; i.e., $u\left(p_{n_{j}}\right) \rightarrow u(p)$ for all $u \in B$. It follows that $\varphi(u)=u(p)$ for all $u \in B^{+}\left(p_{0}\right)$. Hence $p$ is a bounded function on $B^{+}\left(p_{0}\right)$; i.e., $u(p) / u\left(p_{0}\right) \leqq M$ for all $u \in B^{+}\left(p_{0}\right)$, hence for all $u>0$, and $p \in X\left(p_{0}\right)$.

If $D\left(p_{n}, p\right) \rightarrow 0$, then $u\left(p_{n}\right) \rightarrow u(p)$ (uniformly) for $u \in B^{+}\left(p_{0}\right)$, and hence $u\left(p_{n}\right) \rightarrow u(p)$ for all $u \in B$. Therefore $p_{n} \rightarrow p$ in $\mathscr{T}$ if $D\left(p_{n}, p\right) \rightarrow 0$, and $\mathscr{T}_{D} \supset \mathscr{T}$. The family $B^{+}\left(p_{0}\right)$ is $D$-equicontinuous on $X\left(p_{0}\right)$ by definition of $D$, and hence is $\mathscr{T}$-equicontinuous if $\mathscr{T}=\mathscr{T}_{D}$. Conversely, if $B^{+}\left(p_{0}\right)$ is $\mathscr{T}$-equicontinuous on $X\left(p_{0}\right)$, then $\mathscr{T}$ convergence implies $D$-convergence.

We assume for the rest of this section that $X$ is separable (hence $X-\Gamma$ is separable), and that $B$ is a Dirichlet space. Then the representing measures are unique, and $B \mid \Gamma$ is dense in $L_{1}(m)$ (and these are in fact exactly the two conditions we use). If $p \in X\left(p_{0}\right)$, then $m_{p}=K_{p} m$, where $m$ represents $p_{0}$, and $0 \leqq K_{p} \leqq M\left(p, p_{0}\right)$ a.e. $(m)$.

Definition. If $B$ is Dirichlet, and $m$ represents $p_{0}$, let $\mathscr{T}_{\infty}$ be the $L_{\infty}(m)$ metric topology transferred to $X\left(p_{0}\right)$; i.e., let $\|p-q\|_{\infty}=$ $\left\|K_{p}-K_{q}\right\|_{\infty}$.

Theorem 7. If $B$ is Dirichlet and $p, q \in X\left(p_{0}\right)$, then $\|p-q\|_{\infty}=$ $D(p, q)$.

Proof. By definition, $\|p-q\|_{\infty}=\left\|K_{p}-K_{q}\right\|_{\infty}$ is the sup of the integrals $\int u\left(K_{p}-K_{q}\right) d m$, where $u$ runs over the unit ball of $L_{1}(m)$. Since $B$ is Dirichlet, we can take the sup over the functions $u \in B$ with $\int|u| d m=1$, and moreover, we can consider only functions $u$ in $B$ which are strictly positive or strictly negative. Hence $\|p-q\|_{\infty}$ is the sup of $u(p)-u(q)$ for $u \in B$ with $\pm u \in B^{+}\left(p_{0}\right)$, or $\|p-q\|_{\infty}=$ $\sup \left\{|u(p)-u(q)|: u \in B^{+}\left(p_{0}\right)\right\}=D(p, q)$. 
Corollary 1. $\left\{K_{p}: p \in X\left(p_{0}\right)\right\}$ is a closed set in $L_{x}(m)$.

Proof. $\quad D$ is complete.

Corollary 2. $\mathscr{T}=\mathscr{T}_{D}=\mathscr{T}_{\infty}$ on $X\left(p_{0}\right)$ if and only if $B^{+}\left(p_{0}\right)$ is equicontinuous on $X\left(p_{0}\right)$.

Since $p \rightarrow K_{p}$ is in particular a continuous map into $L_{1}(m)$, there is a jointly measurable function $Q(p, \theta)$ on $X\left(p_{0}\right) \times \Gamma$ such that $Q(p, \cdot)=K_{p}$ (in $\left.L_{1}(m)\right)$ [4, Lemma 3.10]. Since we have in fact a homeomorphism $p \rightarrow K_{p}$ into $L_{x}(m)$, we get the stronger result below (cf. [5, Theorem 4]).

THEOREM 8. If $B^{+}\left(p_{0}\right)$ is equicontinuous on $X\left(p_{0}\right)$, and $X$ is separable, and $B$ is Dirichlet, then there is a jointly measurable function $Q(p, \theta)$ on $X\left(p_{0}\right) \times \Gamma$ such that $Q(\cdot, \theta)$ is continuous on $X\left(p_{0}\right)$ for each $\theta \in \Gamma$, $0 \leqq Q(p, \theta) \leqq M\left(p, p_{0}\right) \quad$ for all $(p, \theta) \in X\left(p_{0}\right) \times \Gamma$, and $u(p)=$ $\int u(\theta) Q(p, \theta) d m(\theta)$ for all $u \in B$, all $p \in X\left(p_{0}\right)$.

Proof. By hypothesis, the topology in $X\left(p_{0}\right)$ is given by the $L_{x}(m)$ metric $\|p-q\|_{\infty}$. Let $S$ be countable dense subset of $X\left(p_{0}\right)$. For each $p \in S$, let $Q(p, \cdot)$ be a measurable function defined everywhere on $\Gamma$ such that $Q(p, \cdot) m=m_{p}$. Then

$$
0 \leqq Q(p, \cdot) \leqq M\left(p, p_{0}\right)
$$

and

$$
|Q(p, \cdot)-Q(q, \cdot)| \leqq\|p-q\|_{\infty}
$$

hold a.e. $(m)$ for all $p, q \in S$. Let $E$ be the countable union of zero measure sets where the inequalities above fail, and redefine all $Q(p, \theta)$ to be 1 for $p \in S, \theta \in E$. Now the inequalities above hold for all $p, q \in S$ and all $\theta \in \Gamma$. For $p \in X\left(p_{0}\right)$, define $Q(p, \theta)=\lim Q\left(p_{n}, \theta\right)$ for any sequence $\left\{p_{n}\right\}$ in $S$ converging to $p$. Clearly $Q(p, \cdot)$ is measurable, and $Q(p, \cdot) m$ represents $p$. Since $Q$ is continuous in $p$ for each fixed $\theta, Q$ is jointly measurable.

Corollary. $M\left(p, p_{0}\right)$ is a continuous function of $p$ on $X\left(p_{0}\right)$.

Proof. $M\left(p, p_{0}\right)=\sup \{Q(p, \theta): \theta \in \Gamma\}$.

As in [5], we let $\hat{B}$ denote the closure of $B \mid X\left(p_{0}\right)$ in the topology of uniform convergence on compact subsets of $X\left(p_{0}\right)$. The proofs of the remaining results of [5] are true in our present setting, and the function 
$Q(\cdot, \theta) \in \hat{B}$ except possibly for a $\theta$ in a set of measure zero [5, proof of Theorem 7]. Hence we have the following.

Theorem 9. If $X$ is separable, $B$ is Dirichlet, and $B^{+}\left(p_{0}\right)$ is equicontinuous, then there is a function $Q(p, \theta)$ as in Theorem 8 so that $Q(\cdot, p) \in \hat{B}$ for all $\theta \in \Gamma$.

The main result of [4] gives an integral kernel representation like the above for function spaces which are determined by a family of local integral kernels. For any such space the set $B^{+}\left(p_{0}\right)$ will be equicontinuous on $X\left(p_{0}\right)$ [4, Theorem 1.4], and hence Theorem 9 above will give the end result of [4] for points in $X\left(p_{0}\right)$. The result of [4] holds at all points of $X$, and hence without reference to the ordering $\leqslant$. In the present setting the proofs are simpler, and we naturally obtain more information about the kernel $Q(p, \theta)$.

The classical Herglotz theorem states that: (i) if $v$ is harmonic on the unit ball, then there is a positive measure $\alpha$ on the boundary such that $v(p)=\int P(p, \theta) d \alpha(\theta)$, where $P(p, \theta)$ is the Poisson kernel; and (ii) any function $v$ given by such an integral is harmonic on the open ball. The Herglotz theorem extends to general function spaces with the appropriate hypotheses.

Let $\mathscr{B}^{+}\left(p_{0}\right)$ be the limits of positive functions in $B$ in the sense of uniform convergence on compact subsets of $X\left(p_{0}\right)$. The functions in $\mathscr{B}^{+}\left(p_{0}\right)$ are defined only on $X\left(p_{0}\right)$. If $B$ is the space of continuous functions on $X$ which are harmonic on $X-\Gamma$, and $p_{0} \in X-\Gamma$, then $\mathscr{B}^{+}\left(p_{0}\right)$ is the set of all positive harmonic functions on $X-\Gamma$. If $B$ is the space of continuous functions on $X$ which satisfy the heat equation on $X-\Gamma$ (for appropriate $X-$ cf. $\S 4$ ), then $\mathscr{B}^{+}\left(p_{0}\right)$ is the space of all positive parabolic functions on $X-\Gamma$ if $p_{0}$ is a maximal point of $X-\Gamma$ with respect to $\leqslant$.

If $B^{+}\left(p_{0}\right)$ is equicontinuous, then part (i) of the Herglotz theorem holds on $X\left(p_{0}\right)$ with our kernel $Q(p, \theta)$ in place of the Poisson kernel. If in addition the kernels $Q(p, \cdot)$ are continuous, then part (ii) of the Herglotz theorem holds.

THEOREM 10. If $B^{+}\left(p_{0}\right)$ is equicontinuous, and $\alpha$ is a positive measure on $\Gamma$, and $v(p)=\int Q(p, \theta) d \alpha(\theta)$, then $v \in \mathscr{B}^{+}\left(p_{0}\right)$.

Proof (cf. [7, Theorem 6]). Assume without loss that $\alpha(\Gamma)=1$. The integral can be approximated at any finite number of points of $X\left(p_{0}\right)$ by a finite sum of the form 


$$
F(p)=\Sigma Q\left(p, \theta_{\imath}\right) \alpha\left(\Gamma_{\imath}\right)
$$

where $\left\{\Gamma_{i}\right\}$ is a partition of $\Gamma$. Hence $v$ is the pointwise limit on $X\left(p_{0}\right)$ of functions $F$ of the form (1). Each such function $F$ is in $B^{+}\left(p_{0}\right)$, since $Q\left(p_{0}, \cdot\right) \equiv 1$. The set of functions (1) is equicontinuous on $X\left(p_{0}\right)$, since

$$
\begin{aligned}
\left|F(p)-F\left(p^{\prime}\right)\right| & \leqq \Sigma\left|Q\left(p, \theta_{\imath}\right)-Q\left(p^{\prime}, \theta_{\imath}\right)\right| \alpha\left(\Gamma_{\imath}\right) \\
& \leqq\left\|Q(p, \cdot)-Q\left(p^{\prime}, \cdot\right)\right\|_{\infty} \Sigma \alpha\left(\Gamma_{\imath}\right) \\
& =\left\|Q(p, \cdot)-Q\left(p^{\prime}, \cdot\right)\right\|_{\infty} .
\end{aligned}
$$

The topology in $X\left(p_{0}\right)$ is the same as the $L_{x}(m)$ topology on the kernels $Q(p, \cdot)$, so $\left|F(p)-F\left(p^{\prime}\right)\right|<\epsilon$ for all $F$ of the form (1) if $\left\|p-p^{\prime}\right\|_{\infty}<\epsilon$, and the functions (1) are equicontinuous. The pointwise limit of equicontinuous functions is uniform on compact sets.

THEOREM 11. If $B^{+}\left(p_{0}\right)$ is equicontinuous, and the functions $Q(p, \cdot)$ are continuous on $\Gamma$ for $p \in X\left(p_{0}\right)$, then any function $v$ in $\mathscr{B}^{+}\left(p_{0}\right)$ can be written $v(p)=\int Q(p, \theta) d \alpha(\theta)$ for some positive measure $\alpha$.

Proof. Assume without loss that $v\left(p_{0}\right)=1$, and let $u_{n} \rightarrow v$ u.c.c. on $X\left(p_{0}\right)$, where $u_{n} \in B^{+}\left(p_{0}\right)$. The measures $u_{n} d m$ are in $T$, since

$$
\begin{aligned}
1=u_{n}\left(p_{0}\right) & =\int u_{n}(\theta) Q\left(p_{0}, \theta\right) d m(\theta) \\
& =\int u_{n}(\theta) d m(\theta) .
\end{aligned}
$$

Let $\alpha$ be the $w^{*}$ limit of a subnet $u_{n} d m$. Then since $Q(p, \cdot)$ is continuous,

$$
v(p)=\lim u_{n_{l}}(p)=\lim \int Q(p, \theta) u_{n_{l}}(\theta) d m(\theta)=\int Q(p, \theta) d \alpha(\theta) .
$$

4. Solutions of the heat equation. Let $\varphi_{1}, \varphi_{2}$ be two continuously differentiable functions on $[a, b]$, with $\varphi_{1}(t)<\varphi_{2}(t)$, and let $X=\left\{(x, t): \varphi_{1}(t) \leqq x \leqq \varphi_{2}(t), a \leqq t \leqq b\right\}$. We consider the space $B_{p}$ of continuous functions on $X$ which satisfy the heat equation $u_{x x}(x, t)=$ $u_{t}(x, t)$ in the interior and on the top line: $t=b, \varphi_{1}(b)<x<\varphi_{2}(b)$. We will call $B_{p}$ the space of parabolic functions on $X$.

The Shilov boundary of $B_{p}$ in $X$ consists of the two curves $x=\varphi_{i}(t)$, and the bottom segment $t=a, \varphi_{1}(a) \leqq x \leqq \varphi_{2}(a)[13$, p. 338], and $B \mid \Gamma=C(\Gamma)^{\prime}[13$, p. 354 et. seq. $]$. 
Pini [14] and Hadamard [9] have proved the following Harnack inequality for the parabolic functions: if $\left(x_{1}, t_{1}\right),\left(x_{2}, t_{2}\right) \in X-\Gamma$, then $\left(x_{1}, t_{1}\right) \leqslant\left(x_{2}, t_{2}\right)$ if $t_{1} \leqq t_{2}$, and $M\left(x, t ; x_{2}, t_{2}\right)$ is uniformly bounded for $(x, t)$ in a compact subset of $X\left(x_{2}, t_{2}\right)$. Hence the Gleason parts of $X$ are the singletons of points in $\Gamma$, and the open horizontal segments $P\left(t_{0}\right)=\left\{\left(x, t_{0}\right)\right.$ : $\left.\varphi_{1}\left(t_{0}\right)<x<\varphi_{2}\left(t_{0}\right)\right\}$. The non-singleton parts $P(t)$ are simply ordered by $\leqslant: P\left(t_{1}\right) \leqslant P\left(t_{2}\right)$ if $t_{1} \leqq t_{2}$. If $p_{0}$ is any point of the top segment $P(b)$, then $X\left(p_{0}\right)=X-\Gamma$, and $B^{+}\left(p_{0}\right)$ is equicontinuous. This last follows from the existence of local integral kernels [10], and the fact that the functions of $B^{+}\left(p_{0}\right)$ are uniformly bounded on compact subsets of $X-\Gamma$ (cf. [4, Theorem 1.4]).

Nirenberg [12] has proved various maximum principles for parabolic partial differential equations and inequalities, including the following result for $B_{p}$. If $u \in B_{p}$ and $u\left(x_{0}, t_{0}\right)=\max u$ for some $\left(x_{0}, t_{0}\right) \notin \Gamma$, then $u$ is constant for $t \leqq t_{0}$. That is, $\left(x_{0}, t_{0}\right)$ maximizes $(x, t)$ for all $t \leqq t_{0}$. This of course follows from the Harnack inequality by Theorem 3 .

It follows from the maximum principle that the support of $m_{p}$, where $p=\left(x_{0}, t_{0}\right)$, is a subset of $\Gamma\left(t_{0}\right)=\Gamma \cap\left\{(x, t): t \leqq t_{0}\right\}$. In fact sp $m_{p}=\Gamma\left(t_{0}\right)$ as we show next.

\section{THEOREM 12. If $p=\left(x_{0}, t_{0}\right)$, then $\operatorname{sp} m_{p}=\Gamma\left(t_{0}\right)$.}

Proof. If there is an interval in $\Gamma\left(t_{0}\right)-s p m_{p}$, then we can find a continuous function $u \geqq 0$ so that $u>0$ at points of $\Gamma\left(t_{0}\right)-s p m_{p}$. Hence $u(q)>0$ for some $q \leqslant p, u \geqq 0$, and $u(p)=0$, a contradiction.

The following result now follows from Theorem 9, and the preceding observations.

THEOREM 13. Let $m$ represent any point $\left(x_{0}, b\right)$ of the top segment $P(b)$. There is a jointly measurable function $Q(x, t ; \theta)$ on $(X-\Gamma) \times \Gamma$ such that: $Q(x, t ; \cdot) m$ represents $(x, t) ; Q(\cdot, \cdot ; \theta)$ satisfies the heat equation for each $\theta \in \Gamma ; 0 \leqq Q(x, t ; \theta) \leqq M\left(x, t ; x_{0}, b\right)$; the set of functions $Q(x, t ; \cdot)$ for $(x, t) \in X-\Gamma$ is uniformly closed in the bounded functions on $\Gamma ; Q(x, t ; \theta)=0$ for $\theta \geqq t$ (obvious notational abuse); $Q(x, t ; \cdot)$ is continuous for $\theta=t$.

Proof. The last statement follows from the fact that $Q(x, t ; \theta)$ converges to $Q\left(x, t_{0}, \theta\right)$ uniformly in $\theta$ as $t \rightarrow t_{0}$, and $Q(x, t, \theta) \equiv 0$ for $\theta \geqq t$.

The author conjectures that $Q(x, t ; \theta)$ can be taken to be a continuous function of $\theta$. At any rate the kernel $Q(p, \cdot)$ is continuous on $\Gamma$ when $X$ is a rectangular domain [10]. Hence we have the following analogue of Herglotz Theorem for parabolic functions (cf. [10, p. 373]). 
TheOrem 14. Let $X=[-1,1] \times[0,1], p_{0}=(0,1)$, and let $\Gamma$ be the lower boundary: $\Gamma=\{-1\} \times[0,1] \cup[-1,1] \times\{0\} \cup\{1\} \times[0,1]$. A positive function $v(x, t)$ on $X-\Gamma$ satisfies the heat equation $v_{x x}=v_{t}$ if and only if there is a positive measure $\alpha$ on $\Gamma$ such that $v(x, t)=\int Q(x, t ; \theta) d \alpha(\theta)$.

Proof. The result is essentially a specialization of Theorems 10 and 11. The set $B^{+}\left(p_{0}\right)$ is equicontinuous $[14$, p. 428; 4 , Theorem 1.4]. The kernel $Q(x, t ; \cdot)$ is continuous on $\Gamma[10]$. Each positive solution of the heat equation on $X-\Gamma=(-1,1) \times(0,1)$ is the u.c.c. limit of solutions which are continuous on $X$. To see this last, observe that $v\left(c x, c^{2}(t-1)+\right.$ 1 ) is parabolic on $X-\Gamma$, continuous on $X$ for $0<c<1$, and approaches $v(x, t)$ as $c \rightarrow 1$.

5. Solutions of the wave equation. Let $X$ be the closed triangle bounded by the $x$-axis and the two lines $y= \pm x+1$. Then $(x, y) \in X$ if and only if $y \geqq 0$ and $-1 \leqq x-y \leqq x+y \leqq 1$. Let $B_{h}$ be the space of $C^{2}$ functions on $X$ which satisfy the wave equation: $u_{x x}(x, y)$ $u_{y y}(x, y)=0$. Then $B_{h}$ consists [11, p. 92] of exactly those functions $u$ which can be written $u(x, y)=\frac{1}{2}[G(x-y)+G(x+y)]$ for some $G$ with $G^{\prime \prime}$ continuous on $[0,1]$.

Since $u(x, y) \leqq \max G(x)=\max u(x, 0)$, every function $u \in B_{h}$ assumes its maximum on $\Gamma=[-1,1] \times\{0\}$. Since $B \mid \Gamma$ is uniformly dense in $C(\Gamma)$, no smaller set than $\Gamma$ will serve as a boundary, and hence $\Gamma$ is the Shilov boundary.

The representing measure for $p=\left(x_{0}, y_{0}\right)$, with $y_{0}>0$, is the average of the point masses at $\left(x_{0}-y_{0}, 0\right)$ and $\left(x_{0}+y_{0}, 0\right)$. We will call the points $\left(x_{0} \pm y_{0}, 0\right)$ the support points of $\left(x_{0}, y_{0}\right)$.

To characterize the relation $q \leqslant p$, we first look at the maximum principle ( $p$ maximizes $q$ ), which is a necessary condition. Since $p$ maximizes $q$ if and only if the support point(s) of $q$ are a subset of the support points of $p$, we have the following result.

THEOREM 15. For $B_{h},\left(x_{0}, y_{0}\right)$ maximizes $(x, y)$ if and only if $(x, y)=\left(x_{0}, y_{0}\right)$ or $(x, y)=\left(x_{0}-y_{0}, 0\right)$ or $(x, y)=\left(x_{0}+y_{0}, 0\right)$.

Although the above theorem is the natural form of the maximum principle for $B_{h}$ in terms of the ordering, the following consequence has a more satisfying form.

Corollary 1. If $u(x, y)=\max u$ for all $(x, y)$ in a horizontal segment $S\left(y_{0}\right)=\left\{(x, y) \in X: y=y_{0}\right\}$, then $u$ is constant for $y \geqq y_{0}$. If $u(x, y) \equiv \max u$ on $S\left(y_{0}\right)$ with $y_{0} \leqq \frac{1}{2}$, then $u$ is constant on $X$. 
Proof. If $u(x, y)=\max u$ on $S\left(y_{0}\right)$, then $u$ is constant on all the support points of points in $S\left(y_{0}\right)$. Every point $(x, y)$ with $y \geqq y_{0}$ will have its support points in this set. If $y_{0} \leqq \frac{1}{2}$, the support points of points in $S\left(y_{0}\right)$ constitute all of $\Gamma$.

By Theorem 15 the only possibilities for $(x, y) \leqslant\left(x_{0}, y_{0}\right)$ are $\left(x_{0}, y_{0}\right)$ itself and its two support points. The point masses at $\left(x_{0}-y_{0}, 0\right)$ and $\left(x_{0}+y_{0}, 0\right)$ are absolutely continuous with respect to their average, with Radon-Nikodym derivative bounded by 2 . Hence we have the following.

THEOREM 16. For $B_{h}, q \leqslant p=\left(x_{0}, y_{0}\right)$ if and only if $q=p$ or $q$ is one of the support points $\left(x_{0} \pm y_{0}, 0\right)$ of $p$. If $q<p, M(q, p)=2$.

Corollary 1 (Harnack convegence theorem). Monotone convergence at $\left(x_{0}, y_{0}\right)$ implies convergence at $\left(x_{0} \pm y_{0}, 0\right)$.

COROllary 2. Let $\left\{u_{n}\right\}$ be a monotone sequence of functions in $B_{h}$. If $\left\{u_{n}(x, y)\right\}$ converges for all $(x, y)$ in a horizontal segment $S\left(y_{0}\right)$, then $\left\{u_{n}(x, y)\right\}$ converges for all $(x, y)$ with $y \geqq y_{0}$. If $\left\{u_{n}\right\}$ converges on $S\left(y_{0}\right)$ with $y_{0} \leqq \frac{1}{2}$, then $\left\{u_{n}\right\}$ converges on $X$. If $\left\{u_{n}\right\}$ converges uniformly on $S\left(y_{0}\right)$, then $\left\{u_{n}\right\}$ converges uniformly for $y \geqq y_{0}$, or uniformly on all of $X$ if $y_{0} \leqq \frac{1}{2}$.

Proof. If $\left\{u_{n}\right\}$ converges on a subset $E$ of $\Gamma$, then clearly $\left\{u_{n}\right\}$ converges at every point $(x, y)$ whose support points are in $E$. Uniform convergence of a positive sequence (let $v_{n}=u_{n}-u_{1}$ ) on $S\left(y_{0}\right)$ implies uniform convergence on the support points of points in $S\left(y_{0}\right)$, and hence uniform convergence on the appgopriate subset of $X$.

\section{REFERENCES}

1. H. S. Bear, A geometric characterization of Gleason parts, P.A.M.S., 16 (1965), 407-412.

2. A strict maximum theorem for one-part function spaces and algebras, Bull. Amer. Math. Soc., 70 (1964), 642-643.

3. Lectures on Gleason Parts, Lecture Notes in Mathematics No. 121, Springer Verlag, New York, 1970.

4. H. S. Bear and A. M. Gleason, A glolal integral representation for abstract harmonic functions, J. Math. Mech., 16 (1967), 639-654.

5. H. S. Bear and Bertram Walsh, Integral kernel for one-part function spaces, Pacific J. Math., 23 (1967), 209-215.

6. H. S. Bear and M. L. Weiss, An intrinsic metric for parts, P.A.M.S., 18 (1967), 812-817.

7. H. S. Bear, An Abstract potential theory with continuous kernel, Pacific J. Math., 14 (1964), 407-420.

8. A. M. Gleason, Function algebras, Seminar on Analytic Functions, vol. 2, Institute for Advanced Study, Princeton, 1957. 
9. J. Hadamard, Extension à l'équation de la chaleur d'un théorème de A. Harnack, Rend. del Circ. Mat. di Palermo, (2), 3 (1954), 337-346.

10. Philip Hartman and Aurel Wintner, On the solutions of the equation of heat conduction, Amer. J. Math., 72 (1950), 367-395.

11. Fritz John, Partial Differential Equations, Springer Verlag, New York, 1971.

12. L. Nirenberg, A strong maximum principle for parabolic equations, Comm. on Pure and Appl. Math., 6 (1953), 167-177.

13. I. G. Petrovskii, Partial Differential Equations, W. B. Saunders Co., Philadelphia, 1967.

14. B. Pini, Sulla soluzione generalizzata di Wiener per il primo problema di valori al contorno nel caso parabolico, Rend., Sem. Mat. Univ. Padua, 23 (1954), 422-434.

15. Murray H. Protter and Hans F. Weinberger, Maximum Principles in Differential Equations, Prentice Hall, Englewood Cliffs, New Jersey, 1967.

Received August 6, 1975.

UNIVERSITY OF HAWAII 



\section{PACIFIC JOURNAL OF MATHEMATICS}

\section{EDITORS}

RICHARD ARENS (Managing Editor)

University of California

Los Angeles, California 90024

\section{J. Dugundu}

Department of Mathematics University of Southern California Los Angeles, California 90007

D. Gilbarg and J. Milgram Stanford University Stanford, California 94305

\section{ASSOCIATE EDITORS}
E. F. BECKENBACH
B. H. NeumanN
F. WOLF
K. YoSHIDA

\section{SUPPORTING INSTITUTIONS}

UNIVERSITY OF BRITISH COLUMBIA CALIFORNIA INSTITUTE OF TECHNOLOGY

UNIVERSITY OF CALIFORNIA

MONTANA STATE UNIVERSITY

UNIVERSITY OF NEVADA

NEW MEXICO STATE UNIVERSITY

OREGON STATE UNIVERSITY

UNIVERSITY OF OREGON

OSAKA UNIVERSITY

\author{
UNIVERSITY OF SOUTHERN CALIFORNIA \\ STANFORD UNIVERSITY \\ UNIVERSITY OF HAWAII \\ UNIVERSITY OF TOKYO \\ UNIVERSITY OF UTAH \\ WASHINGTON STATE UNIVERSITY \\ UNIVERSITY OF WASHINGTON \\ AMERICAN MATHEMATICAL SOCIETY
}

The Supporting Institutions listed above contribute to the cost of publication of this Journal, but they are not owners or publishers and have no responsibility for its contents or policies.

Mathematical papers intended for publication in the Pacific Journal of Mathematics should be in typed form or offset-reproduced (not dittoed), double spaced with large margins. Underline Greek letters in red, German in green, and script in blue. The first paragraph or two must be capable of being used separately as a synopsis of the entire paper. Items of the bibliography should not be cited there unless absolutely necessary, in which case they must be identified by author and Journal, rather than by item number. Manuscripts, in duplicate, may be sent to any one of the four editors. Please classify according to the scheme of Math. Reviews, Index to Vol. 39. All other communications should be addressed to the managing editor, or Elaine Barth, University of California, Los Angeles, California, 90024.

100 reprints are provided free for each article, only if page charges have been substantially paid. Additional copies may be obtained at cost in multiples of 50 .

The Pacific Journal of Mathematics is issued monthly as of January 1966. Regular subscription rate: $\$ 72.00$ a year (6 Vols., 12 issues). Special rate: $\$ 36.00$ a year to individual members of supporting institutions.

Subscriptions, orders for back numbers, and changes of address should be sent to Pacific Journal of Mathematics, 103 Highland Boulevard, Berkeley, California, 94708.

PUBLISHED BY PACIFIC JOURNAL OF MATHEMATICS, A NON-PROFIT CORPORATION

Printed at Jerusalem Academic Press, POB 2390, Jerusalem, Israel.

$$
\begin{gathered}
\text { Copyright } 1976 \text { Pacific Journal of Mathematics } \\
\text { All Rights Reserved }
\end{gathered}
$$




\section{Pacific Journal of Mathematics}

Vol. 62, No. 2

February, 1976

Allan Russell Adler and Catarina Isabel Kiefe, Pseudofinite fields, procyclic

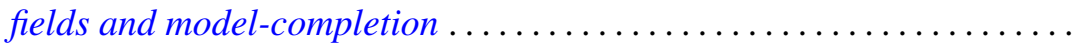

Christopher Allday, The stratification of compact connected Lie group

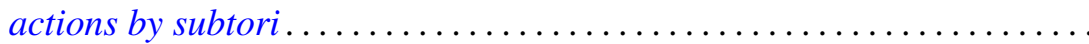

Martin Bartelt, Commutants of multipliers and translation operators .......

Herbert Stanley Bear, Jr., Ordered Gleason parts ..................

James Robert Boone, On irreducible spaces. II .....................

James Robert Boone, On the cardinality relationships between discrete

collections and open covers ............................

L. S. Dube, On finite Hankel transformation of generalized functions .......

Michael Freedman, Uniqueness theorems for taut submanifolds . . . . . . . . .

Shmuel Friedland and Raphael Loewy, Subspaces of symmetric matrices

containing matrices with a multiple first eigenvalue .............

Theodore William Gamelin, Uniform algebras spanned by Hartogs

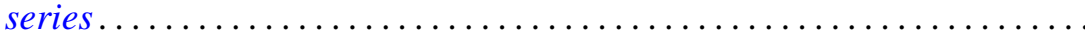

James Guyker, On partial isometries with no isometric part ............

Shigeru Hasegawa and Ryōtarō Satō, A general ratio ergodic theorem for

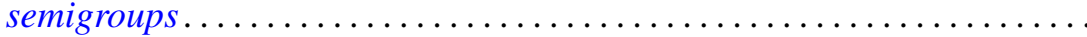

Nigel Kalton and G. V. Wood, Homomorphisms of group algebras with norm less than $\sqrt{2}$.

Thomas Laffey, On the structure of algebraic algebras...

Will Y. K. Lee, On a correctness class of the Bessel type differential operator $S_{\mu}$

Robert D. Little, Complex vector fields and divisible Chern classes ....

Kenneth Louden, Maximal quotient rings of ring extensions . .

Dieter Lutz, Scalar spectral operators, ordered $l^{\rho}$-direct sums, and the

counterexample of Kakutani-McCarthy . .

Ralph Tyrrell Rockafellar and Roger Jean-Baptiste Robert Wets, Stochastic

convex programming: singular multipliers and extended duality

singular multipliers and duality.

Edward Barry Saff and Richard Steven Varga, Geometric overconvergence of rational functions in unbounded domains ..........

Joel Linn Schiff, Isomorphisms between harmonic and P-harmonic Hardy

spaces on Riemann surfaces.

Virinda Mohan Sehgal and S. P. Singh, On a fixed point theorem of

Krasnoselskii for locally convex spaces.

Lewis Shilane, Filtered spaces admitting spectral sequence operations

Michel Smith, Generating large indecomposable continua . 